\title{
Use of $\left[{ }^{18}\right.$ F]Fluoro-2-deoxy-D-glucose Positron Emission Tomographic Imaging in the National Lung Screening Trial
}

Viswam S. Nair, MD; Vandana Sundaram, MPH; Michael K. Gould, MD; and Manisha Desai, PhD

\begin{abstract}
BACKGROUND: Positron emission tomography (PET) is a diagnostic tool for lung cancer evaluation. No studies have ascertained practice patterns and determined the appropriateness of PET imaging in a large group of US patients with screen-detected lung nodules.

METHOdS: We analyzed participants in the National Lung Screening Trial (NLST) with positive screening test results and identified individuals with a PET scan performed prior to lung cancer diagnosis (diagnostic PET). Appropriate scan was defined as one performed in a patient with a nodule $\geq 0.8 \mathrm{~cm}$. Logistic regression was used to assess factors associated with diagnostic PET scan use and appropriateness of PET scan use.
\end{abstract}

RESULTS: Diagnostic PET imaging was performed in 1,556 of 14,195 patients (11\%) with positive screen results; 331 of these $(21 \%)$ were inappropriate. PET scan use by endemic fungal disease area was comparable although patients from the Northeast/Southeast were twice as likely as the West to have a diagnostic PET. Trial arm, older age, sex, nodule size $\geq 0.8 \mathrm{~cm}$, upper lobe location, and spiculated margin were variables positively associated with use. Trial arm, older age, and spiculated margin were positively associated with appropriate use. Only 561 diagnostic PETs $(36 \%)$ were recommended by a radiologist and 284 PETs performed for nodules $<0.8 \mathrm{~cm}(86 \%)$ were ordered despite no recommendation from a radiologist.

CONCLUSIONS: PET imaging was differentially used in the NLST and inappropriately used in many cases against radiologist recommendations. These data suggest PET imaging may be overused in the lung cancer screening population and may contribute to excess health-care costs.

CHEST 2016; 150(3):621-630

KEY WORDS: $\left[{ }^{18} \mathrm{~F}\right]$ Fluoro-2-deoxy-D-glucose positron emission tomography; lung cancer; National Lung Screening Trial; positron emission tomography; screening

ABBREVIATIONS: CDAS $=$ Cancer Database Access System; $\mathrm{CXR}=$ chest radiograph; FDG $=\left[{ }^{18} \mathrm{~F}\right]$ fluoro-2-deoxy-D-glucose; $\mathrm{NCI}=$ National Cancer Institute; NLST $=$ National Lung Screening Trial; $\mathrm{PET}=$ positron emission tomography; SPN = solitary pulmonary nodule

AFFILIATIONS: From the Departments of Medicine and Radiology (Dr Nair) and the Quantitative Sciences Unit (Dr Desai and Ms Sundaram), Stanford University School of Medicine, Stanford, CA; and the Department of Research and Evaluation (Health Services Research and Implementation Science) (Dr Gould), Kaiser Permanente Southern California, Pasadena, CA.
FUNDING/SUPPORT: Dr Nair was sponsored by a LUNGevity Career Development Award.

CORRESPONDENCE TO: Manisha Desai, PhD, Quantitative Sciences Unit, Stanford University School of Medicine, Stanford, CA 94305; e-mail: manisha.desai@stanford.edu

Copyright (C) 2016 American College of Chest Physicians. Published by Elsevier Inc. All rights reserved.

DOI: http://dx.doi.org/10.1016/j.chest.2016.05.006 
$\left[{ }^{18}\right.$ F $]$ Fluoro-2-deoxy-D-glucose positron emission tomography (FDG-PET) imaging was introduced into clinical practice 15 years ago for the diagnosis of solitary pulmonary nodules (SPNs), based on decision models that suggested it was an accurate and cost-effective imaging test. ${ }^{1}$ Meta-analyses shortly thereafter confirmed that FDG-PET imaging was a potentially specific and sensitive tool for both SPN diagnosis and lung cancer staging. ${ }^{2-4}$

The National Lung Screening Trial (NLST) was a prospective, multicenter, randomized trial of lung cancer screening by chest radiography (CXR) vs chest CT scan in patients 55 to 74 years of age with a recent or current smoking history of at least 30 pack-years. The NLST demonstrated that older, high-risk patients with an at least 30 pack-year smoking history who underwent yearly CT scans compared with CXR over 3 years had a decreased lung cancer-specific mortality (relative risk reduction, 20\%). ${ }^{5}$ Comparatively, this is more efficacious than mammography or colon cancer screening. ${ }^{6,7}$ While impressive, the NLST results came with recognition that $96 \%$ of identified lesions, where a "positive" screen result was defined as a nodule $\geq 4 \mathrm{~mm}$ in diameter, were not cancer. ${ }^{5}$ Many of these patients with false positive findings were subjected to additional downstream testing, including imaging in the majority of cases, and invasive biopsies or surgeries in a minority of cases. Despite these additional tests, current cost-effectiveness analyses suggest that lung cancer screening may be effective by modern cost metrics, ${ }^{8}$ but there is strong interest within the medical community to minimize the morbidity and cost of screening by identifying the highest risk patients using mathematical modeling, or blood biomarker integration.

At present, the American College of Chest Physicians, the National Cancer Center Network, and the American College of Radiology recommend that clinicians consider using FDG-PET imaging to risk-stratify indeterminate SPNs of adequate size for downstream management. ${ }^{10,11}$ For the American College of Chest Physicians, these recommendations have decreased in emphasis from 2007 (grade 2a) to the present (grade 2c), which reflects a more stringent appraisal of the quality of evidence and risk of bias. ${ }^{10,12}$ Practice patterns, however, remain poorly characterized at a population level despite the rapidly increasing use of PET scans, with an estimated 2,000 imaging stations in the United States alone. ${ }^{13}$ Since FDG-PET imaging is costly, how it is used in screen-detected nodules for patients who have a positive-or a false positive-result will undoubtedly affect health-care costs and use.

Several European studies have previously examined PET scan use in a lung cancer screening population, but on a much smaller scale when compared with the US-based NLST. ${ }^{14-16}$ We now add to this literature a secondary analysis of a large number of patients who had a positive screen result ( $\geq 4$-mm nodule) and subsequently underwent a diagnostic PET scan to ascertain the etiology of an indeterminate finding prior to a diagnosis in the NLST. Our goal was to characterize diagnostic FDG-PET scan use for screen-detected nodules.

\section{Methods}

\section{Data Sources}

The NLST enrolled more than 54,000 patients from 2002 to 2004 at 33 medical centers. ${ }^{5,17}$ Follow-up for diagnostic evaluation and treatment was left to the enrollment centers and was therefore not standardized. Thus, the NLST was a reflection of medical center practice patternsthe majority of which were academic-for nodule evaluation in addition to a lung cancer screening trial. The NLST was a collaboration between the National Cancer Institute (NCI) and the American College of Radiology Imaging Network (ACRIN). The NCI maintains the Cancer Database Access System (CDAS), which has developed a repository of data dictionaries and patient-level data files for participants in the NLST. These data were posted to the website in locked form in 2012 and consist of 16 de-identified SAS files that are available for statistical analysis. We reviewed variables from these NLST data sets and extracted pertinent variables for our analysis. The CDAS also provided to us at request additional unblinded center locations for regional analyses and to examine the effect of endemic fungal disease on PET scan use (e-Appendix 1). Data specific to individual centers were blinded for publication at the request of the NCI.
All patient data were de-identified and received from the CDAS after (1) proposal approval and (2) material transfer agreements had been signed as part of this institutional review board-exempt study at the Stanford University School of Medicine.

\section{Study Cohort and Variable Definitions}

We selected all patients who had a positive finding on any of the three annual screening scans in either group (Fig 1). A positive finding was defined as a nodule coded as "Positive, Change Unspecified, nodule(s) $\geq 4 \mathrm{~mm}$ or enlarging nodule(s), mass(es), other nonspecific abnormalities suspicious for lung cancer"; "Positive, No Significant Change, stable abnormalities potentially related to lung cancer, no significant change since prior screening exam"; or "Positive, other" in the participant data set from the NLST files. This corresponds to a code of 4,5 , or 6 in the participant identification data dictionary, which specifies the finding of a new $\geq 4$-mm lesion requiring further evaluation.

We assessed whether patients had undergone PET or PET-CT imaging (defined in the data sets as "Radionuclide scan-FDG-PET scan" or "Radionuclide scan-Fusion PET/CT scan"). We defined a diagnostic PET scan as a scan that was performed after a positive finding on a 


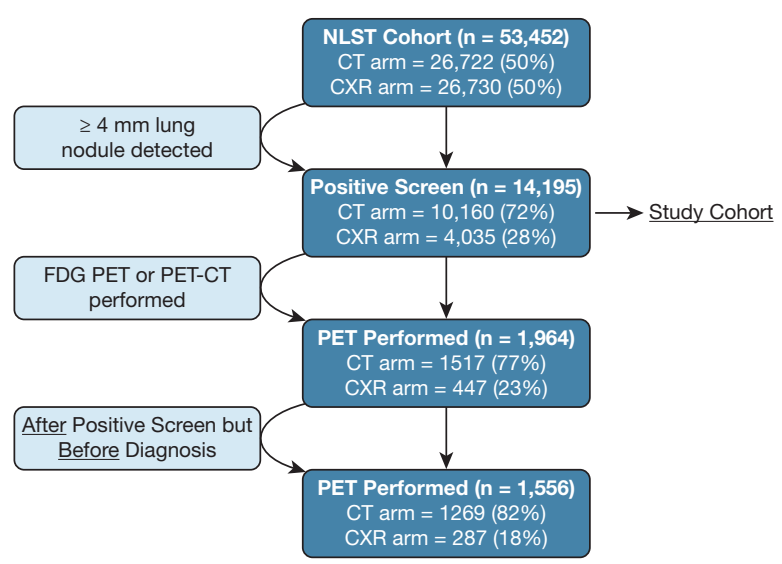

Figure 1 - Use of $\left[{ }^{18} F\right]$ fluoro-2-deoxy-D-glucose positron emission tomography (FDG-PET) in the National Lung Screening Trial (NLST). Shown are patient selection criteria and information on FDG-PET imaging use for the study cohort derived from the NLST. Patients were included in the analytic study cohort from either arm of the NLST if they had any positive screen result (defined as $\geq 0.4-\mathrm{cm}$ lung nodule) during year 1, 2, or 3. We included either FDG-PET or PET-CT scans and defined a diagnostic PET scan as one performed most closely after a positive screen result on CT imaging or chest radiography but before lung cancer diagnosis; for patients without a lung cancer diagnosis, a diagnostic PET scan was defined as the PET scan done soonest after the first positive screen result. $C X R=$ chest radiograph

screening scan and prior to lung cancer diagnosis that was associated with a positive finding; for patients who had not received a diagnosis of lung cancer, we defined the PET scan done after the first positive finding as a diagnostic PET. Thus, the first PET scan performed after a positive finding leading to a work-up represented a diagnostic PET scan for this study.

We determined the number of nodules and nodule characteristics (size, location, border) for each patient's nodules detected from the positive finding associated with the diagnostic PET scan. For nodules without a PET scan, we used the nodules associated with the first positive finding to define nodule characteristics. Nodule size was determined on the basis of the maximum diameter in any dimension (axial or perpendicular). For patients in whom multiple nodules were detected, we used the largest nodule to determine nodule size, location, and border. We stratified patients into one of three groups based on nodule size $(<0.8 \mathrm{~cm}, \geq 0.8-2 \mathrm{~cm},>2 \mathrm{~cm})$. We chose $<0.8 \mathrm{~cm}$ as our lower threshold, based on current recommendations that suggest PET imaging is not useful for nodules smaller than this, given its limited spatial resolution. ${ }^{18}$ We defined appropriateness as PET imaging performed for nodules $\geq 0.8 \mathrm{~cm}$. We categorized nodule location as right upper lobe, left upper lobe, or other, and nodule border as spiculated, smooth, other, or not applicable (not a noncalcified nodule or mass).

We grouped centers into four regions (Northeast, South, Midwest, and West) for analysis. Each enrollment center was categorized by the presence or absence of endemic mycoses based on data provided from the CDAS (e-Table 1). Other variables of interest included age, sex, race/ethnicity, smoking status at baseline, lung cancer diagnosis, death, and whether the PET scan had been recommended by a radiologist.

We also determined the time (in days) from a positive finding to the diagnostic PET scan, and we report the median and interquartile range of this distribution given its skewness.

\section{Statistical Analysis}

The primary outcome was use of diagnostic PET imaging among patients with a positive finding. Secondary outcomes were diagnostic PET imaging among patients with a large nodule $(\geq 0.8 \mathrm{~cm})$, whether PET imaging was recommended by a radiologist after a positive finding, and the appropriateness of PET scan use among patients who underwent diagnostic PET imaging. We examined patient demographics, nodule characteristics, and other variables among patients with and without diagnostic PET imaging. Logistic regression techniques were used to characterize associations. To address our primary objective, we fit univariable and multivariable logistic regression models to determine factors associated with the use of diagnostic PET imaging. We included the following features in the multivariable models: NLST trial arm (CT vs CXR), age at baseline, race/ethnicity, sex, smoking status at baseline, region, nodule size, nodule location, nodule border, and PET imaging recommended by radiologist. We used similar techniques to assess our secondary objectives. Specifically, we employed multivariable logistic regression models to describe associations between patient and nodule characteristics and our secondary outcomes. To account for site-by-site heterogeneity, we included and evaluated a fixed effect for geographic region. We also conducted sensitivity analyses that accounted for clustering by institution by including a random effect for institution in all our models.

The sample size for some of the multivariable models was slightly smaller than the number of subjects eligible for study simply because a subject may have been missing data on at least one variable included in the model. The actual sample sizes used are noted in the tables. All tests were two-sided and conducted at the .05 significance level.

\section{Results}

The study cohort included 14,195 patients who had a positive finding on any screening scan, including 10,160 with a positive CT scan result and 4,035 with a positive CXR result (Fig 1). Of these patients, 1,964 (14\%) underwent a PET scan performed after a positive finding, but only a subset of these-1,556 (11\% of all patients with a positive finding)-received a diagnostic PET scan. Of the 1,556 patients who underwent diagnostic PET imaging, 493 (32\%) received a subsequent diagnosis of cancer, and for 1,063 (68\%) the diagnosis was benign. Of these patients with a malignant lesions, $359(73 \%)$ received a diagnosis of stage I or II cancer.

The median time from a positive finding on CT scan or CXR to diagnostic PET scan was 64 days (interquartile range, 36-319 days). Patients with and without a diagnostic PET scan were similar with respect to sex, race/ethnicity, and smoking status but differed with respect to age, region of enrollment, nodule size, nodule location, and nodule spiculation (Table 1). A similar table by enrollment arm (CT or CXR) is available in e-Table 2. 
TABLE 1] Study Cohort Characteristics by Diagnostic FDG-PET or PET-CT Imaging Use

\begin{tabular}{|c|c|c|c|}
\hline Variable & $\begin{array}{l}\text { Diagnostic PET Scan } \\
(n=1,556) \\
(11 \%)\end{array}$ & $\begin{array}{l}\text { No Diagnostic PET Scan } \\
(\mathrm{n}=12,639) \\
(89 \%)\end{array}$ & $\begin{array}{c}\text { Total } \\
(\mathrm{N}=14,195) \\
(100 \%)\end{array}$ \\
\hline \multicolumn{4}{|l|}{ Prerandomization } \\
\hline \multicolumn{4}{|l|}{ Patient characteristics } \\
\hline \multicolumn{4}{|l|}{ Trial arm } \\
\hline Low-dose CT & $1,269(81.6)$ & $8,891(70.4)$ & $10,160(71.6)$ \\
\hline Chest radiograph & $287(18.4)$ & $3,748(29.7)$ & $4,035(28.4)$ \\
\hline \multicolumn{4}{|l|}{ Age, y } \\
\hline Mean (SD) & $63.2(5.3)$ & $61.9(5.1)$ & $62.0(5.2)$ \\
\hline $55-64$ & $945(60.7)$ & $8,811(69.7)$ & $9,756(68.7)$ \\
\hline $65-74$ & $611(39.3)$ & $3,824(30.3)$ & $4,435(31.3)$ \\
\hline Male (vs female) & $885(56.9)$ & $7,564(59.6)$ & $8,449(59.5)$ \\
\hline \multicolumn{4}{|l|}{ Race/ethnicity ${ }^{a}$} \\
\hline White & $1,409(90.6)$ & $11,461(90.7)$ & $12,870(90.7)$ \\
\hline Black & $62(4.0)$ & $495(4.0)$ & $557(4.0)$ \\
\hline Asian & $27(1.7)$ & $228(1.8)$ & $255(1.8)$ \\
\hline Other & $47(3.0)$ & $385(3.0)$ & $432(3.0)$ \\
\hline Former smoker (vs current) & $758(48.7)$ & $6,379(50.5)$ & $7,137(50.3)$ \\
\hline \multicolumn{4}{|l|}{ Enrollment center characteristics } \\
\hline \multicolumn{4}{|l|}{ Region } \\
\hline Midwest & $574(36.9)$ & $5,814(46.0)$ & $6,388(45.0)$ \\
\hline Northeast & $346(22.2)$ & $1,912(15.1)$ & $2,258(15.9)$ \\
\hline Southeast & $435(28.0)$ & $2,351(18.6)$ & $2,786(19.6)$ \\
\hline West & $201(12.9)$ & $2,562(20.3)$ & $2,763(19.5)$ \\
\hline Fungal disease endemic (yes) & $1,133(72.8)$ & $8,716(69.0)$ & $9,849(69.4)$ \\
\hline \multicolumn{4}{|l|}{ Postrandomization } \\
\hline \multicolumn{4}{|l|}{ Nodule characteristics } \\
\hline Mean nodules per patient (SD) & $3.4(3.0)$ & $2.9(2.6)$ & $3.0(2.7)$ \\
\hline Median nodule size (IQR) ${ }^{\mathrm{b}-\mathrm{d}}$ & $11(8-17)$ & $7(5-10)$ & $7(5-10)$ \\
\hline$<0.8 \mathrm{~cm}$ & $331(21.3)$ & $7,065(55.9)$ & $7,396(52.1)$ \\
\hline$\geq 0.8$ to $2 \mathrm{~cm}$ & $928(59.6)$ & $4,017(31.8)$ & $4,945(34.8)$ \\
\hline$>2.0 \mathrm{~cm}$ & $214(13.8)$ & $541(4.3)$ & $755(5.3)$ \\
\hline \multicolumn{4}{|l|}{ Nodule location ${ }^{\mathrm{b}-\mathrm{d}}$} \\
\hline Right upper lobe & $454(29.2)$ & $2,614(20.7)$ & $3,068(21.6)$ \\
\hline Left upper lobe & $280(18.0)$ & $1,658(13.1)$ & $1,938(13.7)$ \\
\hline Not upper lobe & $739(47.5)$ & $7,351(58.2)$ & $8,090(57.0)$ \\
\hline \multicolumn{4}{|l|}{ Nodule border ${ }^{\text {b-e }}$} \\
\hline Spiculated (stellate) & $442(28.4)$ & $1,040(8.2)$ & $1,482(10.4)$ \\
\hline Smooth & $595(38.2)$ & $6,885(54.5)$ & $7,480(52.7)$ \\
\hline Lung cancer diagnosed (yes) & $493(31.7)$ & 749 (5.9) & $1,242(8.7)$ \\
\hline \multicolumn{4}{|l|}{ FDG-PET characteristics } \\
\hline $\begin{array}{l}\text { PET recommended by radiologist after } \\
\text { positive screen result (size) }\end{array}$ & $561(36.1)$ & $399(3.2)$ & $960(6.8)$ \\
\hline$<0.8 \mathrm{~cm}$ & $47(8.4)$ & $60(15.0)$ & $107(11.2)$ \\
\hline$\geq 0.8$ to $2 \mathrm{~cm}$ & $412(73.4)$ & $250(62.7)$ & $662(69.0)$ \\
\hline$>2.0 \mathrm{~cm}$ & $93(16.6)$ & $59(14.8)$ & $152(15.8)$ \\
\hline Not reported & $9(1.6)$ & $30(7.5)$ & $39(4.1)$ \\
\hline
\end{tabular}

(Continued) 


\begin{tabular}{c|c|c|c}
\hline & $\begin{array}{c}\text { Diagnostic PET Scan } \\
(\mathrm{n}=1,556) \\
(11 \%)\end{array}$ & $\begin{array}{c}\text { No Diagnostic PET Scan } \\
(\mathrm{n}=12,639) \\
(89 \%)\end{array}$ & $\begin{array}{c}\text { Total } \\
(\mathrm{N}=14,195) \\
(100 \%)\end{array}$ \\
\hline $\begin{array}{c}\text { Noriable PET recommended by radiologist } \\
\text { after positive screen result (size) }\end{array}$ & $995(63.9)$ & $12,240(96.8)$ & $13,235(93.2)$ \\
$<0.8 \mathrm{~cm}$ & $284(28.5)$ & $7,005(57.2)$ & $7,289(55.1)$ \\
$\geq 0.8$ to $2 \mathrm{~cm}$ & $516(51.9)$ & $3,767(30.8)$ & $4,283(32.4)$ \\
$>2.0 \mathrm{~cm}$ & $121(12.2)$ & $482(3.9)$ & $603(4.6)$ \\
$\quad$ Not reported & $74(7.4)$ & $986(8.1)$ & $1,060(8.0)$ \\
\hline \hline
\end{tabular}

FDG-PET $=\left[{ }^{18}\right.$ F $]$ fluoro-2-deoxy-D-glucose positron emission tomography; $\mathrm{IQR}=$ interquartile range; NR = not reported; PET = positron emission tomography.

aEleven and 70 patients are missing from the PET and no-PET arms, respectively.

bonly reported for noncalcified nodule or mass; for low-dose CT, the mass had to be $\geq 0.4 \mathrm{~cm}$ in diameter.

'Selected nodules for positive screen result associated with PET scan or associated with first positive screen result in the no-PET arm.

${ }^{d} n=83$ and $n=1,016$ not reported in PET and no-PET arms, respectively.

${ }^{e} n=436$ and $n=3,698$ (unable to determine in PET and no-PET arms), respectively.

Only 552 diagnostic PET scans (35\%) performed after a positive finding were recommended by a radiologist. Of 1,142 diagnostic PET scans, $73 \%$ were performed for patients with nodules $\geq 0.8 \mathrm{~cm}$, and of these, 505 (44\%) were recommended by a radiologist (Table 1$)$. However, 331 diagnostic PET scans (21\%) were performed for patients who had nodules $<0.8 \mathrm{~cm}$; of these only 47 (14\%) were recommended by radiologists (Fig 2).

The Southeast region comprised 10 enrollment centers, the Northeast region comprised nine centers, the Midwest comprised nine centers, and the West comprised five centers (Fig 3). The rate of use varied,

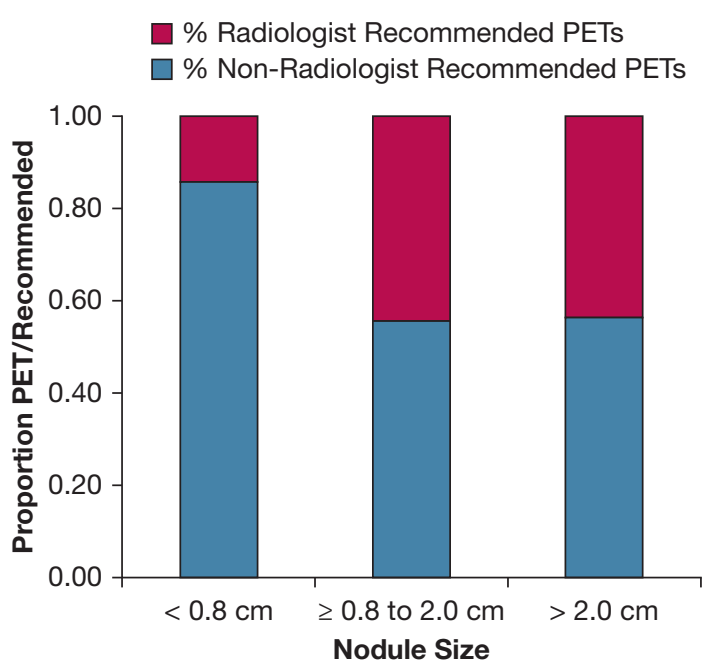

Figure 2 - Practitioner recommendation for diagnostic PET scan by nodule size. Diagnostic PET scan use by nodule size is shown along with the proportion of these PET scans that were recommended by a radiologist. It can be seen that radiologists were more conservative in recommending PET imaging for small nodules. See Figure 1 legend for expansion of abbreviation. from $4 \%$ to $29 \%$ by individual center and by region from $9 \%$ in the West to $17 \%$ in the Northeast. For patients with nodules $\geq 0.8 \mathrm{~cm}$, we noted nonsignificant variations in appropriate PET imaging use across the 33 centers, ranging from $8 \%$ to $43 \%$, and from $17 \%$ in the West to $26 \%$ in the Northeast (Fig 4). Although PET imaging use was similar in areas with and without endemic fungal disease, patients in the Northeast (OR, 2.3; 95\% CI, 1.9-2.8) and Southeast (OR, 2.4; 95\% CI, 2.0-2.8) regions were twice as likely to undergo diagnostic PET imaging as patients in the West (Table 2, Univariable column).

Being in the CT trial arm, older, female, from a region other than the West, having a PET scan recommendation by a radiologist, a nodule size $\geq 0.8$, or an upper lobe or spiculated nodule were all associated with higher odds of diagnostic PET imaging use after a positive finding (Table 2, Multivariable column). In particular, a radiology recommendation (OR, 8.2; 95\% CI, 7.0-9.7) and a nodule size $>2 \mathrm{~cm}$ (OR, 5.0; 95\% CI, 3.9-6.3) demonstrated large associations with diagnostic PET imaging use after adjusting for covariates. When PET scan recommendation by a radiologist was removed from the model the results were similar, with large nodule size having a similarly high magnitude of association (data not shown). When we restricted our analysis to those patients who had a nodule $\geq 0.8 \mathrm{~cm}$, we found similar results (Table 2, Multivariable [Nodule $\geq 0.8 \mathrm{~cm}$ ] column).

Being in the CT trial arm, older, female, from the Northeast, or from an area with endemic fungal disease, having a nodule in the right upper lobe, with a size $\geq 0.8 \mathrm{~cm}$, or a nodule with spiculated or smooth features were characteristics associated with increased 


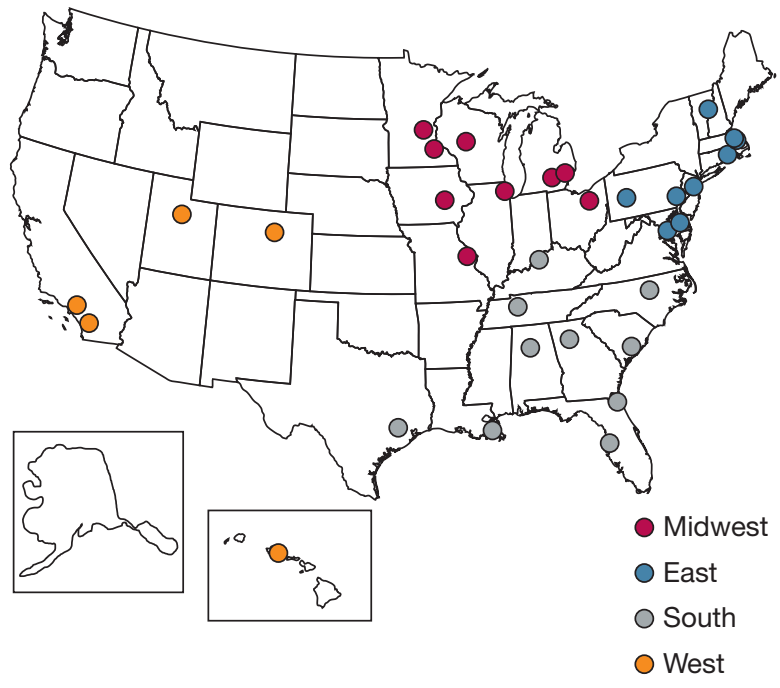

Figure 3 - Enrollment centers by region in the NLST. The 33 centers in the NLST were distributed across geographically diverse areas of the United States, with various types of endemic fungal disease (e-Table 1). We grouped these 33 centers into four regions for further analysis. See Figure 1 legend for expansion of abbreviation.

odds of a PET scan recommendation by a radiologist as a follow-up to a positive finding (Table 3 ).

Being older (adjusted OR, 1.05; 95\% CI, 1.02-1.07) and having a nodule with spiculated margins (adjusted OR,

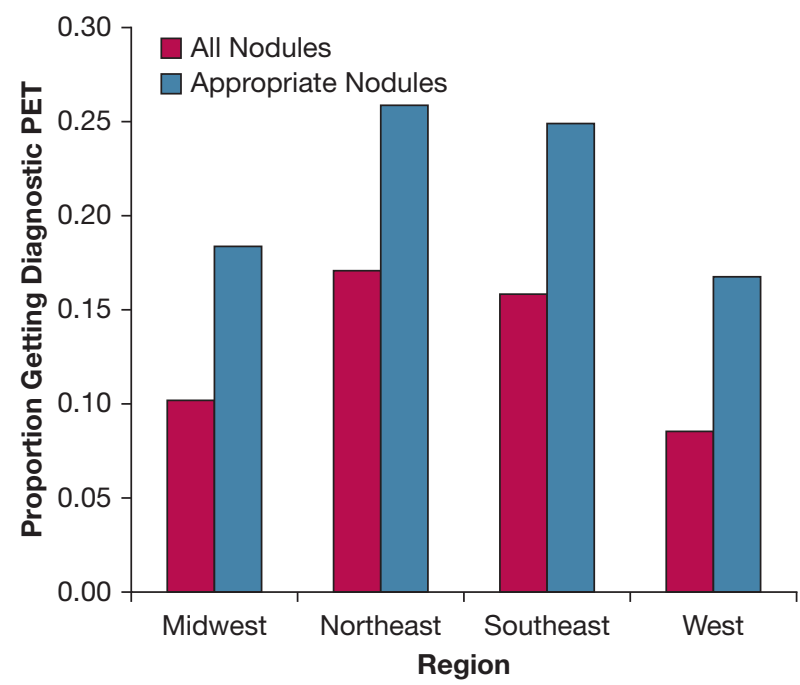

Figure 4 - Diagnostic PET imaging use by region. Diagnostic FDG PET and PET-CT imaging use grouped by region (Figure 3) is shown stratified by appropriate use (ie, PET imaging use in all nodules and those in $\geq 0.8-\mathrm{cm}$ nodules only). We observed nonsignificant variations in individual center and regional use for both groups (see the Results section). Regional differences were significantly associated with use for all nodules whether appropriately imaged or not, however (Table 2). Note: For appropriateness, 83 were missing information because nodule size not available. See Figure 1 legend for expansion of abbreviations.
1.6; 95\% CI, 1.1-2.5) were associated with higher odds of having an appropriate PET scan whether or not a radiologist recommended the test (Table 4). Being in the CT arm was associated with lower odds of having an appropriate PET scan as compared with being in the chest radiograph arm (adjusted OR, 0.4; 95\% CI, 0.3-0.6).

A logistic regression examining factors associated with appropriate PET imaging, using a threshold of $\geq 1.0 \mathrm{~cm}$ for nodule appropriateness, found that trial arm, age, and nodule margins remained significantly associated with appropriate PET imaging; however, age was no longer significantly associated with appropriate PET imaging. A PET scan recommendation by a radiologist continued to show the strongest association with appropriate PET imaging (OR, 3.8; 95\% CI, 2.9-5.0).

In our sensitivity analysis that accounted for clustering by institution, the association between geographic region and outcome decreased. For example, in our multivariable model of factors associated with PET imaging use after a positive screen result (Table 2, third column), only the Northeast still had significantly higher, but diminished, odds of PET imaging use compared with the West (OR, 1.4; 95\% CI, 1.2-2.7; $P<.01)$. We found a similar result when restricting our analysis to patients with nodules $\geq 0.8 \mathrm{~cm}$ (Table 2 , fourth column: Northeast (OR, 1.6; 95\% CI, 1.1-2.5) (compared with West). However, in our analysis of factors associated with PET scan recommendation by a radiologist after a positive screen result (Table 3), there was no statistically significant difference by region.

\section{Discussion}

This is the first study to examine the use of diagnostic PET imaging of screen-detected lung nodules for a large cohort of patients in the United States. Our findings of documented practice patterns from 2002 to 2004 identify areas for improvement in implementing lung cancer screening care for abnormal imaging. Other studies have examined PET imaging use for lung nodule diagnosis in areas with endemic mycoses ${ }^{19}$ and by survey of clinical practice patterns. ${ }^{20}$ However, the first study was a meta-analysis of heterogeneous cohorts of non-screen-detected lung nodules and the second was an observational study consisting of only 377 patients. Importantly, our study corroborates a similar finding in a retrospective study of US veterans at 15 medical centers, where guideline-inconsistent care for patients with lung nodules was commonplace. ${ }^{21}$ 
TABLE 2 ] Factors Associated With Diagnostic FDG-PET Imaging Use After a Positive Screen Result

\begin{tabular}{|c|c|c|c|}
\hline \multirow[b]{2}{*}{ Variable } & \multirow[b]{2}{*}{$\begin{array}{c}\text { Univariable }^{b} \\
(n=14,195) \\
{[\mathrm{OR}(95 \% \mathrm{CI})]}\end{array}$} & \multicolumn{2}{|c|}{ Multivariable ${ }^{a}$} \\
\hline & & $\begin{array}{l}\text { Study Cohort } \\
(n=13,020) \\
{[\mathrm{OR}(95 \% \mathrm{CI})]}\end{array}$ & $\begin{array}{l}\text { Nodules } \geq 0.8 \mathrm{~cm} \\
\quad(n=5,668) \\
{[\mathrm{OR}(95 \% \mathrm{CI})]}\end{array}$ \\
\hline Trial arm (CT vs CXR) & $1.9(1.6-2.1)$ & $1.8(1.5-2.1)$ & $2.0(1.7-2.4)$ \\
\hline Age & $1.05(1.04-1.06)$ & $1.03(1.02-1.05)$ & $1.04(1.03-1.06)$ \\
\hline Sex (female vs male) & $1.1(1.02-1.3)$ & $1.2(1.02-1.3)$ & $1.1(1.0-1.3)$ \\
\hline \multicolumn{4}{|l|}{ Race/ethnicity (vs white) } \\
\hline Hispanic & $1.2(0.8-1.9)$ & $1.3(0.8-2.1)$ & $1.2(0.6-2.2)$ \\
\hline Black non-Hispanic & $1.0(0.8-1.3)$ & $1.0(0.7-1.3)$ & $1.1(0.7-1.6)$ \\
\hline Asian non-Hispanic & $1.0(0.6-1.4)$ & $1.8(1.1-2.9)$ & $2.0(1.1-3.6)$ \\
\hline Other & $0.8(0.5-1.2)$ & $1.1(0.6-1.8)$ & $1.2(0.6-2.3)$ \\
\hline Smoking status (current vs former) & $1.1(1.0-1.2)$ & $1.0(0.9-1.2)$ & $1.1(0.9-1.3)$ \\
\hline \multicolumn{4}{|l|}{ Region (vs West) } \\
\hline Midwest & $1.3(1.1-1.5)$ & $1.5(1.2-2.0)$ & $1.4(1.1-1.9)$ \\
\hline Northeast & $2.3(1.9-2.8)$ & $2.3(1.9-3.0)$ & $1.8(1.4-2.4)$ \\
\hline Southeast & $2.4(2.0-2.8)$ & $2.1(1.6-2.7)$ & $1.7(1.3-2.2)$ \\
\hline Endemic fungal disease & $1.2(1.1-1.4)$ & $1.0(0.8-1.2)$ & $1.0(0.8-1.2)$ \\
\hline PET recommended by radiologist & $17.3(15.0-20.0)$ & $8.2(7.0-9.7)$ & $7.6(6.4-9.1)$ \\
\hline \multicolumn{4}{|l|}{ Nodule size (vs $<0.8 \mathrm{~cm}$ ) } \\
\hline$\geq 0.8$ to $2 \mathrm{~cm}$ & $4.9(4.3-5.6)$ & $3.3(2.9-3.9)$ & NA \\
\hline$>2 \mathrm{~cm}$ & $8.4(7.0-10.2)$ & $5.0(3.9-6.3)$ & NA \\
\hline \multicolumn{4}{|l|}{ Nodule location (vs not upper lobe) } \\
\hline Left upper lobe & $1.7(1.4-1.9)$ & $1.4(1.1-1.6)$ & $1.5(1.2-1.8)$ \\
\hline Right upper lobe & $1.7(1.5-2.0)$ & $1.3(1.1-1.5)$ & $1.3(1.1-1.5)$ \\
\hline \multicolumn{4}{|l|}{ Nodule margins (vs other) } \\
\hline Spiculated & $3.6(3.1-4.2)$ & $1.7(1.5-2.1)$ & $1.7(1.4-2.1)$ \\
\hline Smooth & $0.7(0.6-0.8)$ & $1.0(0.8-1.1)$ & $1.0(0.7-1.1)$ \\
\hline
\end{tabular}

CXR = chest radiograph; NA = not applicable. See Table 1 legend for expansion of other abbreviations.

aultivariable logistic regression of all variables.

beffect of individual variables on PET imaging use.

The higher use of diagnostic PET in upper lobe, spiculated nodules that were $\geq 0.8 \mathrm{~cm}$ in diameter is consistent with its intended use since these nodules are more likely to be malignant. ${ }^{10,12}$ Our results therefore suggest that providers were using PET imaging appropriately for more concerning nodules that were of at least indeterminate risk.

However, spatial resolution limits the resolution of PET imaging. ${ }^{22}$ Guidelines therefore suggest that nodules $<0.8 \mathrm{~cm}$ in diameter should not be imaged by PET. ${ }^{10,11}$ Almost one-quarter of patients who underwent a diagnostic PET scan had nodules $<0.8 \mathrm{~cm}$ in this analysis. These were recommended by radiologists for only $14 \%$ of these patients, suggesting that radiologists were more conservative in their use of PET imaging compared with other practitioners managing nodules.
Estimates of lung cancer screening candidacy have identified more than 7 million eligible persons in the United States alone, based on NLST criteria. ${ }^{23}$ According to our analyses, 289 PET scans were performed for nodules $<0.8 \mathrm{~cm}$ out of 26,722 total subjects screened in the CT arm in the NLST. This translates to a $1.08 \%$ prevalence of unnecessary PET scans for lung cancer screening by CT. On the basis of this prevalence of "misuse" and given a PET scan cost of US $\$ 2,000,{ }^{24}$ we could therefore potentially decrease medical costs in the United States by $\$ 151$ million when adhering to existing guidelines.

While there was regional variation in PET imaging use that could reflect a differential rate in technology adoption in the NLST enrollment centers, there was no difference in PET imaging use by endemic fungal disease 
TABLE 3 ] Factors Associated With Radiologist Recommendation for FDG-PET Imaging Use After a Positive Screen Result

\begin{tabular}{|c|c|}
\hline Variable & $\begin{array}{c}\text { PET Scan Recommended by } \\
\text { Radiologist } \\
(\mathrm{n}=13,020) \\
{[\mathrm{OR}(95 \% \mathrm{CI})]}\end{array}$ \\
\hline Trial arm (CT vs CXR) & $9.6(7.2-12.6)$ \\
\hline Age & $1.02(1.01-1.04)$ \\
\hline Sex (female vs male) & $1.3(1.2-1.6)$ \\
\hline \multicolumn{2}{|l|}{ Race/ethnicity (vs white) } \\
\hline Hispanic & $2.0(1.1-3.4)$ \\
\hline Black & $0.9(0.6-1.3)$ \\
\hline Asian & $0.8(0.4-1.6)$ \\
\hline Other & $0.7(0.4-1.4)$ \\
\hline $\begin{array}{l}\text { Smoking status (current } \\
\text { vs former) }\end{array}$ & $1.1(0.9-1.3)$ \\
\hline \multicolumn{2}{|l|}{ Region (vs West) } \\
\hline Midwest & $0.4(0.3-0.5)$ \\
\hline Northeast & $1.9(1.5-2.4)$ \\
\hline Southeast & $0.9(0.7-1.1)$ \\
\hline Endemic fungal disease & $2.2(1.8-2.7)$ \\
\hline \multicolumn{2}{|l|}{ Nodule size (vs $<0.8 \mathrm{~cm}$ ) } \\
\hline$\geq 0.8$ to $2 \mathrm{~cm}$ & $12.6(10.1-15.7)$ \\
\hline$>2 \mathrm{~cm}$ & $22.7(16.9-30.4)$ \\
\hline \multicolumn{2}{|l|}{$\begin{array}{l}\text { Nodule location (vs not } \\
\text { upper lobe) }\end{array}$} \\
\hline Left upper lobe & $1.3(1.1-1.6)$ \\
\hline Right upper lobe & $1.4(1.2-1.6)$ \\
\hline \multicolumn{2}{|l|}{ Nodule margins (vs other) } \\
\hline Spiculated & $3.1(2.5-3.7)$ \\
\hline Smooth & $1.4(1.2-1.7)$ \\
\hline
\end{tabular}

Multivariable models use a sample size that is slightly smaller than the number of subjects eligible for study, simply because a subject may have been missing data on at least one variable included in the model. The actual sample sizes used are noted in the tables. See Table 1 and 2 legends for expansion of abbreviations.

region, which can confound the interpretation of PET, as shown by Deppen et al. ${ }^{19}$ In fact, the region that PET was used in most (Northeast) is an area with a lower prevalence of endemic mycosis. That study also confirmed that the initial studies in PET effectiveness for diagnosing SPNs may have been overestimated, especially its sensitivity ( $97 \%$ vs $78 \%$ ), ${ }^{1,3,19}$ which is consistent with generally optimistic estimates of biomarkers during the early phases of research and clinical application. ${ }^{25}$ Further, the inappropriate use of FDG-PET imaging of small nodules during the NLST may have contributed to the assertion of decreased sensitivity of PET in routine clinical use.
Our results suggest significant variation in outcome by region. After performing sensitivity analyses that accounted for clustering by center, similar results for all correlates of interest persisted in all models, with the exception of the finding (not surprisingly) that corresponded to region.

One unexpected finding in this study was that the CT trial arm of the NLST was associated with twice the odds of having undergone a diagnostic PET scan. We do not have a ready explanation for this but believe that usage bias from differential practice patterns could play a role in producing this difference. We are currently exploring this in additional studies.

Our study has several methodologic limitations. First, we combined either PET or PET-CT imaging for the purpose of the study. At the time of this trial from 2002 to 2004, PET-CT imaging had just recently been approved, and there has been a natural shift to PET-CT imaging over time to the present day that may affect the generalizability of our results. Second, the time at which this study was performed may not reflect current practice patterns and increasing awareness (and therefore knowledge) of the appropriate use of PET imaging as a diagnostic tool. Third, our definition of a diagnostic PET scan was based on the diagnosis of cancer, and patients undergoing PET scans prior to a tissue diagnosis to assess extent of disease that were being clinically "staged" may have been misclassified as having a diagnostic-rather than a staging-PET scan.

We also note that increasing awareness of nodule evaluation guidelines may not necessarily translate into improved care since the most recent study examining current physician practice patterns for nodule assessment revealed a $37 \%$ diagnostic PET scan usage rate, ${ }^{20}$ which is much higher than the $11 \%$ we report here from the NLST enrollment era 10 years prior. It is also possible that differences in baseline cancer prevalence may explain this disparity in use.

To determine whether our results held for "NSLT enrollment era practice patterns" we used a cutoff of $\geq 1.0 \mathrm{~cm}$ to define appropriate diagnostic PET imaging based on guideline statements from 2003. ${ }^{26,27}$ On the basis of this threshold, 213 additional patients would have received inappropriate diagnostic PET imaging, for a total of $544(35 \%)$.

Third, we considered a PET scan performed prior to a definitive malignant diagnosis and at any time after a benign positive finding to be a diagnostic PET scan, 
TABLE 4 ] Factors Associated With Appropriate PET Imaging Use Among Patients Who Underwent Diagnostic PET Imaging

\begin{tabular}{|c|c|c|}
\hline Variable & $\begin{array}{c}\text { Appropriate PET Scan } \\
\text { (With Radiologist Recommendation) } \\
(\mathrm{n}=1,462) \\
{[\mathrm{OR}(95 \% \mathrm{CI})]}\end{array}$ & $\begin{array}{c}\text { Appropriate PET Scan } \\
\text { (Excluding Radiologist Recommendation) } \\
(\mathrm{n}=1,462) \\
{[\mathrm{OR}(95 \% \mathrm{CI})]}\end{array}$ \\
\hline Trial arm (CT vs CXR) & $0.4(0.3-0.6)$ & $0.6(0.4-0.9)$ \\
\hline Age & $1.05(1.02-1.07)$ & $1.04(1.02-1.07)$ \\
\hline Sex (female vs male) & $0.8(0.6-1.1)$ & $0.8(0.6-1.1)$ \\
\hline \multicolumn{3}{|l|}{ Race/ethnicity (vs white) } \\
\hline Hispanic & $0.7(0.3-2.1)$ & $0.7(0.3-1.8)$ \\
\hline Black & $2.0(1.0-4.2)$ & $1.7(0.8-3.4)$ \\
\hline Asian & $0.8(0.2-2.5)$ & $0.7(0.2-2.0)$ \\
\hline Other & $1.1(0.3-4.1)$ & $1.3(0.3-4.6)$ \\
\hline Smoking status (current vs former) & $1.0(0.7-1.3)$ & $1.0(0.8-1.4)$ \\
\hline \multicolumn{3}{|l|}{ Region (vs West) } \\
\hline Midwest & $0.8(0.5-1.5)$ & $0.6(0.4-1.04)$ \\
\hline Northeast & $0.5(0.3-0.8)$ & $0.5(0.3-0.9)$ \\
\hline Southeast & $0.7(0.4-1.2)$ & $0.6(0.4-1.02)$ \\
\hline Endemic fungal disease & $0.8(0.6-1.2)$ & $1.1(0.7-1.5)$ \\
\hline PET recommended by radiologist & $5.9(4.1-8.4)$ & $\ldots$ \\
\hline \multicolumn{3}{|l|}{ Nodule location (vs not upper lobe) } \\
\hline Left upper lobe & $1.1(0.7-1.6)$ & $1.1(0.8-1.6)$ \\
\hline Right upper lobe & $1.0(0.7-1.4)$ & $1.1(0.8-1.5)$ \\
\hline \multicolumn{3}{|l|}{ Nodule margins (vs other) } \\
\hline Spiculated & $1.6(1.1-2.5)$ & $1.9(1.2-2.8)$ \\
\hline Smooth & $0.4(0.3-0.5)$ & $0.4(0.3-0.5)$ \\
\hline
\end{tabular}

See Table 1 and 2 legends for expansion of abbreviations.

although we cannot absolutely know how the recommending or ordering provider truly intended its use since PET imaging is now routinely recommended to identify occult regional or distant disease. ${ }^{4,28}$ Last, this was an analysis of US practice patterns, the majority being academic centers, and therefore the generalizability of our findings to the community and to other countries is unclear.

\section{Conclusions}

Lung cancer screening by CT scanning in the United States from 2002 to 2004 resulted in a diagnostic FDG-PET usage rate of $11 \%$ across all participants with a positive screen result in the NLST. Practitioners appropriately used PET imaging for higher risk, older patients with more concerning nodules based on radiographic appearance, but diagnostic PET scans were performed inappropriately almost one in four times for smaller nodules that were below the imaging resolution of PET imaging. There was considerable regional variation in PET imaging use, and our findings suggest that the odds were lower that inappropriate PET imaging was performed when radiologists did not recommend a PET scan. Adherence to existing guidelines put forth by several organizations could lead to more optimal management of patients with screendetected nodules and a potential reduction in costs for the US health-care system. 


\section{Acknowledgments}

Author contributions: V. S. N. and M. D.: concept, design, analysis, writing, and approval; V. S. and M. K. G.: design, analysis, writing, and approval. V. S. N. serves as guarantor.

Financial/nonfinancial disclosures: None declared.

Role of sponsors: The sponsor had no role in the design of the study, the collection and analysis of the data, or the preparation of the manuscript.

Other contributions: The authors thank Josh Rathmell, MS, of the NCI Cancer Data Access System for data release; and the NLST Investigators, the NCI, and the ACRIN for releasing data into the public domain.

Additional information: The e-Tables can be found in the Supplemental Materials section of the online article.

\section{References}

1. Gambhir SS, Shepherd JE, Shah BD, et al. Analytical decision model for the costeffective management of solitary pulmonary nodules. J Clin Oncol. 1998;16(6):2113-2125.

2. Gould MK, Maclean CC, Kuschner WG, Rydzak CE, Owens DK. Accuracy of positron emission tomography for diagnosis of pulmonary nodules and mass lesions: a meta-analysis. JAMA. 2001;285(7):914-924.

3. Gould MK, Sanders GD, Barnett PG, et al. Cost-effectiveness of alternative management strategies for patients with solitary pulmonary nodules. Ann Intern Med. 2003;138(9):724-735.

4. van Tinteren H, Hoekstra OS, Smit EF, et al. Effectiveness of positron emission tomography in the preoperative assessment of patients with suspected non-small-cell lung cancer: the PLUS multicentre randomised trial. Lancet. 2002;359(9315):1388-1393.

5. National Lung Screening Trial Research Team, Aberle DR, Adams AM, et al. Reduced lung-cancer mortality with lowdose computed tomographic screening. N Engl J Med. 2011;365(5):395-409.

6. Humphrey LL, Helfand M, Chan BK, Woolf SH. Breast cancer screening: a summary of the evidence for the U.S. Preventive Services Task Force. Ann Intern Med. 2002;137(5 Part 1):347-360.

7. Ferlitsch M, Reinhart K, Pramhas S, et al. Sex-specific prevalence of adenomas, advanced adenomas, and colorectal cancer in individuals undergoing screening colonoscopy. JAMA. 2011;306(12): 1352-1358.

8. Black WC, Gareen IF, Soneji SS, et al. Cost-effectiveness of CT screening in the National Lung Screening Trial. N Engl J Med. 2014;371(19):1793-1802.

9. de Koning HJ, Meza R, Plevritis SK, et al. Benefits and harms of computed tomography lung cancer screening strategies: a comparative modeling study for the U.S. Preventive Services Task Force. Ann Intern Med. 2014;160(5): 311-320.

10. Gould MK, Donington J, Lynch WR, et al. Evaluation of individuals with pulmonary nodules: when is it lung cancer? Diagnosis and management of lung cancer, 3rd ed: American College of Chest Physicians evidence-based clinical practice guidelines. Chest. 2013;143(5 Suppl):e93S-e120S.

11. Ettinger DS, Akerley W, Borghaei H, et al. Non-small cell lung cancer, version 2.2013. J Natl Compr Canc Netw. 2013; 11(6):645-653.

12. Wahidi MM, Govert JA, Goudar RK, Gould MK, McCrory DC. Evidence for the treatment of patients with pulmonary nodules: when is it lung cancer? ACCP evidence-based clinical practice guidelines (2nd edition). Chest. 2007;132(3 Suppl): 94S-107S.

13. Buck AK, Herrmann K, Stargardt T, Dechow T, Krause BJ, Schreyogg J. Economic evaluation of PET and PET/CT in oncology: evidence and methodologic approaches. J Nucl Med. 2010;51(3): 401-412.

14. Ashraf H, Dirksen A, Loft A, et al. Combined use of positron emission tomography and volume doubling time in lung cancer screening with low-dose CT scanning. Thorax. 2011;66(4):315-319.

15. Bastarrika G, Garcia-Velloso MJ, Lozano MD, et al. Early lung cancer detection using spiral computed tomography and positron emission tomography. Am J Respir Crit Care Med. 2005;171(12):1378-1383.

16. Pastorino U, Bellomi M, Landoni $\mathrm{C}$, et al. Early lung-cancer detection with spiral CT and positron emission tomography in heavy smokers: 2-year results. Lancet. 2003;362(9384):593-597.

17. National Lung Screening Trial Research Team, Aberle DR, Berg CD, et al. The National Lung Screening Trial: overview and study design. Radiology. 2011;258(1): 243-253.
18. Rahmim A, Qi J, Sossi V. Resolution modeling in PET imaging: theory, practice, benefits, and pitfalls. Med Phys. 2013;40(6):064301.

19. Deppen SA, Blume JD, Kensinger CD, et al. Accuracy of FDG-PET to diagnose lung cancer in areas with infectious lung disease: a meta-analysis. JAMA. 2014; 312(12):1227-1236.

20. Tanner NT, Aggarwal J, Gould MK, et al Management of pulmonary nodules by community pulmonologists: a multicenter observational study. Chest. 2015;148(6): 1405-1414.

21. Wiener RS, Gould MK, Slatore CG, Fincke BG, Schwartz LM, Woloshin S. Resource use and guideline concordance in evaluation of pulmonary nodules for cancer: too much and too little care. JAMA Intern Med. 2014;174(6): 871-880.

22. Shankar LK, Hoffman JM, Bacharach S, et al. Consensus recommendations for the use of ${ }^{18}$ F-FDG PET as an indicator of therapeutic response in patients in National Cancer Institute Trials. J Nucl Med. 2006;47(6):1059-1066.

23. Humphrey L, Deffebach M, Pappas M, et al. Screening for Lung Cancer: Systematic Review to Update the U.S. Preventive Services Task Force Recommendation [Evidence Synthesis No. 105]. Rockville, MD: Agency for Healthcare Research and Quality; 2013. AHRQ publication 13-05188-EF-1. http://www.ncbi.nlm.nih. gov/books/NBK154610/pdf/Bookshelf NBK154610.pdf. Published July 2013. Accessed June 15, 2016.

24. Cao JQ, Rodrigues GB, Louie AV, Zaric GS. Systematic review of the costeffectiveness of positron-emission tomography in staging of non-small-cell lung cancer and management of solitary pulmonary nodules. Clin Lung Cancer. 2012;13(3):161-170.

25. Ioannidis JP. Why most published research findings are false. PLoS Med. 2005;2(8):e124.

26. Ost D, Fein AM, Feinsilver SH. Clinical practice: the solitary pulmonary nodule. N Engl J Med. 2003;348(25):2535-2542.

27. Tan BB, Flaherty KR, Kazerooni EA, Iannettoni MD. American College of Chest Physicians. The solitary pulmonary nodule. Chest. 2003;123(1 Suppl): 89S-96S.

28. Fischer B, Lassen U, Mortensen J, et al. Preoperative staging of lung cancer with combined PET-CT. N Engl J Med. 2009;361(1):32-39. 